\title{
Defects in nuclear structure and function promote dilated cardiomyopathy in lamin $\mathbf{A} / \mathrm{C}-$ deficient mice
}

\author{
Vesna Nikolova, ${ }^{1}$ Christiana Leimena, ${ }^{1}$ Aisling C. McMahon, ${ }^{2}$ Ju Chiat Tan, ${ }^{2}$ \\ Suchitra Chandar, ${ }^{1}$ Dilesh Jogia, ${ }^{3}$ Scott H. Kesteven, ${ }^{2}$ Jan Michalicek, ${ }^{1}$ Robyn Otway, ${ }^{1}$ \\ Fons Verheyen, ${ }^{4}$ Stephen Rainer, ${ }^{5}$ Colin L. Stewart, ${ }^{6}$ David Martin, ${ }^{3}$ Michael P. Feneley, ${ }^{2}$ \\ and Diane Fatkin ${ }^{1}$ \\ ${ }^{1}$ Molecular Cardiology Program, \\ ${ }^{2}$ Cardiac Mechanics Program, \\ ${ }^{3}$ Molecular Genetics Program, Victor Chang Cardiac Research Institute, Darlinghurst, New South Wales, Australia \\ ${ }^{4}$ Department of Molecular Cell Biology, Cardiovascular Research Institute Maastricht (CARIM), University of Maastricht, \\ Maastricht, The Netherlands \\ ${ }^{5}$ Division of Anatomical Pathology, St. Vincent's Hospital, Darlinghurst, New South Wales, Australia \\ ${ }^{6}$ Laboratory of Cancer and Developmental Biology, National Cancer Institute-Frederick Cancer Research and \\ Development Center, Frederick, Maryland, USA
}

Laminopathies are a group of disorders caused by mutations in the LMNA gene that encodes the nuclear lamina proteins, lamin A and lamin C; their pathophysiological basis is unknown. We report that lamin A/C-deficient ( $\left.\mathrm{Lmna}^{-/}\right)$mice develop rapidly progressive dilated cardiomyopathy (DCM) characterized by left ventricular $(\mathrm{LV})$ dilation and reduced systolic contraction. Isolated $\mathrm{Lmna}^{-/}$ myocytes show reduced shortening with normal baseline and peak amplitude of $\mathrm{Ca}^{2+}$ transients. $L m n a^{-/-} \mathrm{LV}$ myocyte nuclei have marked alterations of shape and size with central displacement and fragmentation of heterochromatin; these changes are present but less severe in left atrial nuclei. Electron microscopy of $\mathrm{Lmna}^{-/-}$cardiomyocytes shows disorganization and detachment of desmin filaments from the nuclear surface with progressive disruption of the cytoskeletal desmin network. Alterations in nuclear architecture are associated with defective nuclear function evidenced by decreased SREBP1 import, reduced PPAR $\gamma$ expression, and a lack of hypertrophic gene activation. These findings suggest a model in which the primary pathophysiological mechanism in Lmna/mice is defective force transmission resulting from disruption of lamin interactions with the muscle-specific desmin network and loss of cytoskeletal tension. Despite severe DCM, defects in nuclear function prevent $\mathrm{Lmna}^{-/-}$cardiomyocytes from developing compensatory hypertrophy and accelerate disease progression.

J. Clin. Invest. 113:357-369 (2004). doi:10.1172/JCI200419448.

\section{Introduction}

Lamins belong to the intermediate filament family of proteins and are predominant components of the nuclear lamina, a thin protein layer that separates the nuclear envelope from the nuclear matrix. Lamins also

Received for publication July 10, 2003, and accepted in revised form November 4, 2003.

Address correspondence to: Diane Fatkin, Victor Chang Cardiac Research Institute, Level 6, 384 Victoria Street, Darlinghurst, New South Wales 2010, Australia. Phone: 61-2-9295 8518; Fax: 61-2-9295 8501; E-mail: d.fatkin@victorchang.unsw.edu.au. Vesna Nikolova and Christiana Leimena contributed equally to this work.

Conflict of interest: The authors have declared that no conflict of interest exists.

Nonstandard abbreviations used: dilated cardiomyopathy (DCM); electrocardiogram/electrocardiographic (ECG); left ventricle/ventricular (LV); left atrium/atrial (LA); preload recruitable stroke work (PRSW); sterol response element-binding protein-1 (SREBP1); atrial natriuretic peptide (ANP); brain natriuretic peptide (BNP); $\beta$-myosin heavy chain ( $\beta$-MHC); myosin light chain 2v (MLC2v); sarcoplasmic reticulum $\mathrm{Ca}^{2+}$-ATPase 2a (SERCA2a); SREBP-cleavage-activating protein (SCAP). form a thin fibrillar web throughout the nuclear matrix $(1,2)$. Lamins are classified as A type or B type. A-type lamins are widely expressed in differentiated cells and include four transcripts produced by alternate splicing of the LMNA gene: lamins A, C, A $\delta 10$, and C2. B-type lamins are present in embryonic cells and are coexpressed with A-type lamins in differentiated cells. B-type lamins include lamins B1, B2, and B3, which are transcribed from the $L M N B 1$ (B1) and $L M N B 2(\mathrm{~B} 2, \mathrm{~B} 3)$ genes, respectively. Lamins $A, C$, and $B 2$ are present in the adult heart (3). Lamins have a critical role in maintaining the structural integrity of the nuclear lamina and the spatial organization of proteins in the inner nuclear membrane. Interactions with lamins are also required for correct positioning of nuclear pore complexes and for anchoring heterochromatin at the nuclear periphery. Although the role of intranuclear lamins has not been clearly defined, the lamin scaffolding may contribute to the internal stability of the nuclear matrix. Collectively, these functions implicate lamins as important determinants of nuclear architecture (4-6). 
The discovery of mutations in the LMNA gene has highlighted a previously unsuspected role of nuclear lamina proteins in the pathogenesis of a variety of human diseases, including dilated cardiomyopathy (DCM) with conduction system disease (7), EmeryDreifuss muscular dystrophy (8), limb girdle muscular dystrophy type 1B (9), familial partial lipodystrophy $(10,11)$, Charcot-Marie-Tooth disease type 2 (12), mandibuloacral dysplasia (13), Hutchinson-Gilford progeria (14-16), and atypical Werner's syndrome (17). The mechanisms by which LMNA gene mutations cause these diverse tissue-specific phenotypes have not been elucidated.

Three principal hypotheses for the pathogenesis of laminopathies have been proposed (reviewed in refs. 5, 6). First, abnormal interactions of mutant lamins with heterochromatin and with transcription regulators such as the retinoblastoma protein might alter gene expression. Second, structural weakness of the lamina might be a predisposing factor to mechanical stressinduced nuclear damage and apoptosis. Third, mutant lamins might have defective protein interactions with as yet undefined consequences. In vitro studies performed using transfected cells and patient fibroblasts have shown that some LMNA mutations cause focal nuclear envelope defects, mislocalization of mutant and WT lamin A/C, lamin B1, lamin B2, and emerin, and altered susceptibility to heat shock (13, 14, 17-24). While these data indicate that LMNA mutations can perturb nuclear properties, the precise relationship between these changes and the disease process has yet to be determined.

We have focused on the role of lamin $\mathrm{A} / \mathrm{C}$ in the pathogenesis of DCM. Targeted deletion of the murine LMNA gene has been reported previously (25). Homozygous (Lmna ${ }^{-/}$) mice exhibit growth retardation from 2-3 weeks of age with clinical and histological features of muscular dystrophy by 3-4 weeks and uniform death by 8 weeks of age. Agematched heterozygous ( $\mathrm{Lmna}^{+/-}$) mice exhibit no overt abnormalities. Although myocardial tissue sections from $L m n a^{-/-}$mice show focal myocyte degenerative changes, the effects of lamin A/C deficiency on cardiac structure and function in vivo have not been evaluated. Here we show that $\mathrm{Lmna}^{-/-}$mice develop DCM with conduction abnormalities by 4-6 weeks of age. Detailed ultrastructural evaluation of $\mathrm{Lmna}^{-/-}$cardiomyocytes indicates that lamin A/C deficiency primarily causes destabilization of nuclear lamina structure and enhanced nuclear deformability, with intranuclear and extranuclear sequelae that promote the development of a severe form of DCM and limit compensatory hypertrophy.

\section{Methods}

Lmna knockout mice. Lamin A/C-deficient mice were generated by targeted deletion of the Lmna gene, as reported previously (25). Mouse genotypes were determined by PCR amplification of tail genomic
DNA. Mice were maintained and studied according to protocols approved by our institutional Animal Ethics Committee.

Transthoracic echocardiography. Echocardiographic studies were performed in anesthetized mice $(2.5 \%$ avertin, $0.005-0.010 \mathrm{ml} / \mathrm{g}$ ) attached to an electrocardiographic (ECG) monitor using a $12-\mathrm{MHz}$ phased array or $15-\mathrm{MHz}$ linear array (for mice less than $12 \mathrm{~g}$ ) probe and a Sonos 5500 ultrasonograph (Philips Medical Systems, Andover, Massachusetts, USA). Left ventricular (LV) parameters were obtained from M-mode recordings (or two-dimensional images for mice less than $12 \mathrm{~g}$ ) in the short-axis view. Orthogonal left atrial (LA) diameters were obtained from two-dimensional images in the long-axis view.

Micromanometry and sonomicrometry. Mice were ventilated, anesthetized with ketamine (100 mg/kg), xylazine $(20 \mathrm{mg} / \mathrm{kg})$, and atropine $(2.5 \mathrm{mg} / \mathrm{kg})$, and then cannulated via the right common carotid artery with a $1.4 \mathrm{~F}$ micromanometer catheter (Millar Instruments Inc., Houston, Texas, USA), and LV pressure was recorded. After sternotomy, pericardiotomy, and placement of a ligature (5.0 silk) around the inferior vena cava, four ultrasound crystals $(0.7 \mathrm{~mm}$ outside diameter; Sonometrics Corp., London, Ontario, Canada) were secured to the epicardial surface with surgical glue. LV volume was derived from the epicardial baseapex and anterior-posterior dimensions (26). Preload recruitable stroke work (PRSW) was derived from a series of beats over a wide preload range induced by transient vena caval occlusion (27).

Isolated myocyte studies. Cardiac myocytes were isolated as described (28). Cells loaded with the $\mathrm{Ca}^{2+}$-sensitive, single excitation, dual-emission fluorescent indicator Indo-1/AM $(1.2 \mu \mathrm{M})$ were superfused with a standard Krebs-Henseleit solution containing $1.25 \mathrm{mM} \mathrm{Ca}^{2+}$ at $32^{\circ} \mathrm{C}$. Myocyte images were stored and dimensions obtained using Image Tool software. Cell shortening was measured by edge detection at $240 \mathrm{~Hz}$ using a variable frame-rate camera (MyoCam; IonOptix Corp., Milton, Massachusetts, USA). Simultaneous changes in intracellular $\mathrm{Ca}^{2+}$ concentration were monitored (28).

Light microscopy. Animals were sacrificed by cervical dislocation. Excised hearts were blotted dry, weighed, rinsed in PBS, fixed in 4\% paraformaldehyde, and embedded in paraffin blocks. Myocardial sections (4 $\mu \mathrm{m}$ ) were stained with H\&E, Masson's trichrome (for interstitial fibrosis), or colloidal iron (for ECM) and were evaluated using light microscopy.

Immunofluorescence microscopy. Frozen tissue sections were fixed and then incubated with primary goat polyclonal anti-lamin B Ab (1:80 dilution; Santa Cruz Biotechnology Inc., Santa Cruz, California, USA), mouse anti-desmin mAb (1:100 dilution; Novocastra Laboratories Ltd., Newcastle, United Kingdom), rabbit polyclonal anti-sterol response element-binding protein-1 (SREBP1) Ab (1:25 dilution; Santa Cruz Biotechnology Inc.), and FITC-conjugated secondary Ab's. Paraffin-embedded myocardial tissue sections were 
Table 1

Echocardiographic and ECG studies in mice aged 2 weeks and 4-6 weeks

\begin{tabular}{|c|c|c|c|c|c|c|}
\hline & \multicolumn{3}{|c|}{2 weeks } & \multicolumn{3}{|c|}{ 4-6 weeks } \\
\hline & WT & $\mathrm{Lmna}^{+/-}$ & $\mathrm{Lmna}^{-/-}$ & WT & $\mathrm{Lmna}^{+/-}$ & $\mathrm{Lmna}^{-/-}$ \\
\hline $\mathrm{BW}(\mathrm{g})$ & $7.6 \pm 1.6$ & $7.5 \pm 1.0$ & $6.0 \pm 0.3^{A, B}$ & $17.4 \pm 2.8$ & $18.5 \pm 2.7$ & $8.7 \pm 1.6^{\mathrm{A}, \mathrm{B}}$ \\
\hline $\operatorname{LVDD}(\mathrm{mm})$ & $2.63 \pm 0.08$ & $2.70 \pm 0.09$ & $2.65 \pm 0.15^{\mathrm{C}, \mathrm{D}}$ & $2.93 \pm 0.24$ & $2.97 \pm 0.23$ & $3.58 \pm 0.23^{\mathrm{A}, \mathrm{B}}$ \\
\hline $\operatorname{LVSD}(\mathrm{mm})$ & $1.22 \pm 0.05$ & $1.23 \pm 0.03$ & $1.23 \pm 0.03^{\mathrm{C}, \mathrm{D}}$ & $1.11 \pm 0.14$ & $1.22 \pm 0.18$ & $3.17 \pm 0.21^{\mathrm{A}, \mathrm{B}}$ \\
\hline LVFS (\%) & $54 \pm 3$ & $55 \pm 1$ & $54 \pm 3$ & $62 \pm 3$ & $59 \pm 5$ & $11 \pm 3^{\mathrm{A}, \mathrm{B}}$ \\
\hline LVWT (mm) & $0.34 \pm 0.01$ & $0.36 \pm 0.02$ & $0.34 \pm 0.01^{C, D}$ & $0.69 \pm 0.08$ & $0.70 \pm 0.06$ & $0.33 \pm 0.05^{A, B}$ \\
\hline LVWT/(LVDD/2) & $0.26 \pm 0.01$ & $0.27 \pm 0.02$ & $0.26 \pm 0.01$ & $0.47 \pm 0.08$ & $0.48 \pm 0.04$ & $0.19 \pm 0.03^{\mathrm{A}, \mathrm{B}}$ \\
\hline LV mass (mg) & $18.4 \pm 1.4$ & $20.5 \pm 1.2$ & $18.6 \pm 2.8^{C}$ & $55.2 \pm 10.6$ & $58.2 \pm 11.2$ & $30.7 \pm 5.8^{\mathrm{A}, \mathrm{B}}$ \\
\hline $\operatorname{LAD}(\mathrm{mm})$ & $1.33 \pm 0.04$ & $1.35 \pm 0.04$ & $1.34 \pm 0.08^{C, D}$ & $1.51 \pm 0.05$ & $1.53 \pm 0.03$ & $1.51 \pm 0.06^{\mathrm{C}, \mathrm{D}}$ \\
\hline Heart rate (bpm) & $613 \pm 63$ & $632 \pm 50$ & $580 \pm 32$ & $681 \pm 84$ & $686 \pm 38$ & $552 \pm 83^{A, B}$ \\
\hline PR interval (ms) & $31.7 \pm 3.9$ & $30.8 \pm 4.6$ & $32.5 \pm 8.1$ & $30.3 \pm 1.6$ & $28.6 \pm 2.3$ & $37.1 \pm 4.8^{\mathrm{A}, \mathrm{B}}$ \\
\hline QRS duration (ms) & $32.2 \pm 3.5$ & $35.9 \pm 5.7$ & $32.0 \pm 7.4$ & $28.5 \pm 3.6$ & $30.0 \pm 2.2$ & $40.3 \pm 7.4^{\mathrm{A}, \mathrm{B}}$ \\
\hline
\end{tabular}

Cardiac function was evaluated in vivo using transthoracic echocardiography with simultaneous ECG monitoring in mice aged 2 weeks $(n=8$ each group) and 4- 6 weeks ( $n=16$ each group). End-diastolic and end-systolic LV dimensions and end-systolic LA dimensions were obtained. LVFS was derived by: $\left[(\right.$ LVDD - LVSD) $/ L V D D] \times 100$. LV mass was derived by: (anterior LVWT + posterior LVWT + LVDD) ${ }^{3}-$ LVDD $^{3}$. All measurements were averaged from a minimum of three separate cardiac cycles. ${ }^{A} P<0.05$ compared with WT, ${ }^{\mathrm{B} P}<0.05$ compared with $L m n a^{+} /-$(statistically significant differences before normalization for BW); $C P<0.05$ compared with WT, $D P<0.05$ compared with $L m n a^{+/-}$(statistically significant differences only after normalization for BW). BW, body weight; LAD, left atrial diameter; LVDD, LV end-diastolic diameter; LVFS, LV fractional shortening; LVSD, LV end-systolic diameter; LVWT, LV wall thickness (anterior wall data shown).

deparaffinized, permeabilized, fixed, and then hybridized with a mouse pancentromeric heterochromatin probe (protocol G; Cambio Ltd., Cambridge, United Kingdom). Sections were counterstained with DAPI and examined with a Leica fluorescence microscope at a magnification of $\times 40$ (lamin B, desmin), $\times 63$ (SREBP1), or $\times 100$ (centromeric heterochromatin).

Apoptosis detection. For in situ detection of DNA fragmentation, paraffin-embedded myocardial sections were deparaffinized, permeabilized, and triple labeled using a TUNEL assay (Promega Corp., Madison, Wisconsin, USA), DAPI, and mouse anti- $\alpha$ sarcomeric actinin. Sections were examined with a Leica fluorescence microscope at $\times 40$ magnification. DNA fragmentation in LV tissue was also evaluated by an endlabeling method (29).

Electron microscopy. Tissues were fixed in $2.5 \% \mathrm{glu}-$ taraldehyde $/ 0.1 \mathrm{M}$ sodium cacodylate buffer, washed, and postfixed with $2 \%$ osmium tetroxide and $2 \%$ uranyl acetate solution, dehydrated in ethanol series, and embedded in epoxy resin. Sections were viewed by transmission electron microscope (Hitachi 7000) at a magnification of $\times 6,000$. For immunogold labeling, hearts were perfused with $0.5 \%$ glutaraldehyde $/ 2.5 \%$ paraformaldehyde in $0.1 \mathrm{M}$ sodium cacodylate buffer ( $\mathrm{pH}$ 7.2), excised, fixed, and embedded in LR White resin (Polysciences Inc., Warrington, Pennsylvania, USA) as described (30). Tissue sections were placed on 200 mesh nickel grids and immunolabeled with primary mouse anti-desmin $\mathrm{mAb}$ and secondary anti-mouse IgG conjugated with $15-\mathrm{nm}$ gold particles (Amersham Life Sciences Inc., Arlington Heights, Illinois, USA). Immunogold-labeled sections were stained with uranyl acetate and lead citrate and examined by transmission electron microscope at $\times 30,000$ magnification.
RNA analysis. Total RNA was isolated from LV tissue using Tri-Reagent (Invitrogen Life Technologies, Carlsbad, California, USA). Northern blot analyses were performed using $\alpha^{32} \mathrm{P}$ dATP-labeled cDNA or $\gamma^{32} \mathrm{P}$ dATPlabeled oligonucleotide probes for atrial natriuretic peptide (ANP), brain natriuretic peptide (BNP), $\beta$-myosin heavy chain ( $\beta-\mathrm{MHC})$, desmin, myosin light chain $2 \mathrm{v}$ (MLC2v), phospholamban, sarcoplasmic reticulum $\mathrm{Ca}^{2+}$-ATPase 2a (SERCA2a), $\alpha$-skeletal actin, SREBP1, and GAPDH. Hybridization signals were quantified using ImageQuant software (Molecular Dynamics, Sunnyvale, California, USA) and were normalized to the signal intensity of GAPDH. Expression levels of c-fos and c-jun were determined by real-time PCR using the LightCycler FastStart DNA Master SYBR Green I kit (Roche Diagnostics GmbH, Mannheim, Germany) with normalization to $\beta$-actin.

Protein analysis. For Western blot analyses, total protein was extracted from frozen LV tissue, separated on an SDS-PAGE gel, and hybridized with primary mouse anti-desmin $\mathrm{mAb}$ (1:100 dilution; Novocastra Laboratories Ltd.) or rabbit polyclonal anti-PPAR $\gamma \mathrm{Ab}$ (1:1,000 dilution, Affinity Bioreagents Inc., Golden, Colorado, USA) with anti-mouse or anti-rabbit IgG HRP-conjugated secondary Ab's, respectively. Nuclear and cytosolic protein fractions were prepared from fresh LV tissue using NE-PER kits (Pierce Biotechnology Inc., Rockford, Illinois, USA), separated on an SDS-PAGE gel, and hybridized with primary rabbit polyclonal anti-SREBP1 Ab (1:200 dilution; Santa Cruz Biotechnology Inc.) with an anti-rabbit IgG HRPconjugated secondary Ab.

Statistical analysis. All data were analyzed by observers who were blinded to the mouse genotype. Continuous variables were analyzed using ANOVA and Student's $t$ tests or multiple linear regression analysis (PRSW) (31). 
Table 2

LV hemodynamic studies in mice aged 4-6 weeks

\begin{tabular}{lccc}
\hline & WT & Lmna $^{+/-}$ & Lmna $^{-/-}$ \\
Heart rate $(\mathrm{bpm})$ & $362 \pm 58$ & $395 \pm 58$ & $392 \pm 64$ \\
$\mathrm{BW}(\mathrm{g})$ & $20.0 \pm 3.2$ & $20.6 \pm 2.6$ & $8.5 \pm 1.8^{\mathrm{A}, \mathrm{B}}$ \\
Wall volume $(\mu \mathrm{l})$ & $61.0 \pm 10.6$ & $60.1 \pm 7.7$ & $31.4 \pm 6.5^{\mathrm{A}, \mathrm{B}}$ \\
End-diastolic volume $(\mu \mathrm{l})$ & $57 \pm 23$ & $56 \pm 26$ & $41 \pm 9^{\mathrm{C}, \mathrm{D}}$ \\
Wall thickness/radius & $0.40 \pm 0.12$ & $0.43 \pm 0.13$ & $0.27 \pm 0.06^{\mathrm{A}, \mathrm{B}}$ \\
Systolic pressure $(\mathrm{mmHg})$ & $83.0 \pm 13.1$ & $87.4 \pm 12.6$ & $63.4 \pm 6.4^{\mathrm{A}, \mathrm{B}}$ \\
End-diastolic pressure $(\mathrm{mmHg})$ & $3.5 \pm 1.1$ & $4.8 \pm 4.2$ & $2.2 \pm 2.7$ \\
$d P / d t_{\max }(\mathrm{mmHg} / \mathrm{s})$ & $4,417 \pm 982$ & $4,663 \pm 1,666$ & $3,084 \pm 860^{\mathrm{A}, \mathrm{B}}$ \\
$d P / d t_{\min }(\mathrm{mmHg} / \mathrm{s})$ & $-4,864 \pm 1,347$ & $-5,000 \pm 1,506$ & $-3,037 \pm 859^{\mathrm{A}, \mathrm{B}}$ \\
Tau $(\mathrm{ms})$ & $12.07 \pm 1.51$ & $13.75 \pm 3.98$ & $17.35 \pm 6.59$ \\
PRSW slope $(\mathrm{ml} \times \mathrm{mmHg} / \mu \mathrm{l} / \mathrm{g})$ & $1.13 \pm 0.66$ & $1.15 \pm 0.28$ & $0.36 \pm 0.19^{\mathrm{A}, \mathrm{B}}$ \\
PRSW volume intercept $(\mu \mathrm{l} / \mathrm{g})$ & $4.39 \pm 0.71$ & $4.09 \pm 0.78$ & $6.61 \pm 1.10^{\mathrm{A}, \mathrm{B}}$ \\
& & &
\end{tabular}

LV function was assessed in vivo using micromanometry and sonomicrometry in WT $(n=9)$, $\mathrm{Lmna}^{+/-}(n=11)$, and $\mathrm{Lmna}^{-/-}(n=6)$ mice. Six beats of steady-state data were acquired; data were recorded at $1 \mathrm{kHz}$. All LV pressure and volume data are after thoracotomy. LV wall volume was derived by: LV weight/1.05. LV endocardial end-diastolic volume was derived by: $\left[(\pi / 6) \times a \times b^{2}\right]-$ LV wall volume, where $a$ and $b$ are the base-apex and anterior-posterior epicardial dimensions, respectively. ${ }^{A} P<0.05$ compared with $\mathrm{WT},{ }^{\mathrm{B} P}<0.05$ compared with $\mathrm{Lmna}^{+/-}$(statistically significant differences before normalization for BW); ${ }^{C} P<0.05$ compared with WT, D $P<0.05$ compared with $\mathrm{Lmna}^{+/-}$(statistically significant differences only after normalization for BW). BW, body weight.

Statistical comparisons for in vivo studies of cardiac size and function were performed before and after normalization for body weight differences. Since body weight and tibial length are both affected by the disease process in $\mathrm{Lmna}^{-/-}$mice, normalization of data to these standard parameters has limitations. The marked differences in body size between the three groups of mice, however, needs to be taken into account when interpreting chamber dimension data. Data are expressed as mean plus or minus SD.

\footnotetext{
Results

Lmna ${ }^{-/}$mice exbibit cardiac function abnormalities in vivo. At birth, there were no differences in body weight or heart weight between WT, $L m n a^{+/-}$, and $L m n a^{-/-}$mice. By 2 weeks of age, $\mathrm{Lmna}^{-/-}$mice demonstrated growth retardation, with a $50 \%$ reduction in body weight by 4-6 weeks of age when compared with WT and $L m n a^{+/-}$ littermates (Tables 1 and 2, Figure 1). Gross examina-
}

tion showed that heart sizes were similar in the three groups at 4-6 weeks (Figure 1b). In $L m n a^{-/-}$mice, the LV was dilated, globular-shaped, and thin-walled, with a lower mass than in WT and $L m n a^{+/}$mice. This LV remodeling was reflected by a reduction in the wall thickness/chamber radius ratio (Tables 1 and 2, Figure $1 \mathrm{~b}$ ). Echocardiographic studies showed a severe global reduction in LV contraction in $\mathrm{Lmna}^{-/-}$mice at 4-6 weeks of age (Table 1). LV systolic function was further investigated using micromanometry and sonomicrometry to measure the relationship between LV stroke work and enddiastolic volume (termed PRSW), a highly reproducible, load-independent index of contractility (26). LV systolic pressure and heart rates were relatively low in all groups, due to the effects of anesthesia and the thoracotomy procedure (32). The presence of marked LV contractile dysfunction and dilation in $\mathrm{Lmna}^{-/-}$mice was evident from a $70 \%$ reduction in PRSW slope and increased volume intercept (Table 2, Figure 2a) (26). Lmna ${ }^{-1-}$ mice also developed LV diastolic dysfunction with a decrease in the peak negative value of the first derivative of $L V$ pressure $\left(d P / d t_{\min }\right)$. There was a trend toward prolongation of tau, although this did not achieve statistical significance (Table 2). A rightward and downward shift was observed in the LV enddiastolic pressure-volume relationship (Figure 2b). Determination of the stress-strain relationship, which is independent of body size, wall thickness, and chamber geometry, however, showed that myocardial wall stiffness was unchanged (Figure 2c). Cardiac function in $\mathrm{Lmna}^{+/-}$mice was similar to that of WT mice (Tables 1 and 2). ECG parameters were similar in the three groups of mice at 2 weeks. At 4-6 weeks, $L m n a^{-/}$mice had relatively slow heart rates with lengthened PR and QRS intervals (Table 1).

Lamin A/C-deficient myocytes exbibit contractile dysfunction. To determine whether the cardiac dysfunction

\section{Figure 1}

Comparative morphometry in mice aged 4-6 weeks. (a) When compared with WT (left) and $\mathrm{Lmna}^{+/-}$littermates (center), Lmna ${ }^{-/}$(right) mice exhibit growth retardation with $50 \%$ reduction in body weight. (b) Despite marked differences in body size, longitudinal sections show that heart sizes are similar in WT (left), $\mathrm{Lmna}^{+/-}$(center), and Lmna ${ }^{-/-}$(right) mice. Lmna ${ }^{-/-}$ hearts show LV and LA dilation with wall thinning. Scale bar: $2.5 \mathrm{~mm}$. (c) The phenotype of severe DCM without compensatory hypertrophy in 4- to 6-week-old $\mathrm{Lmna}^{-1-}$ mice is reflected by Northern blot analyses that show appropriate increases in LV expression of ANP and BNP but no induction of $\beta$-MHC or $\alpha$-skeletal actin. +/+, WT; +/-, Lmna ${ }^{+/-} ;-/-$, Lmna $^{-/}$.
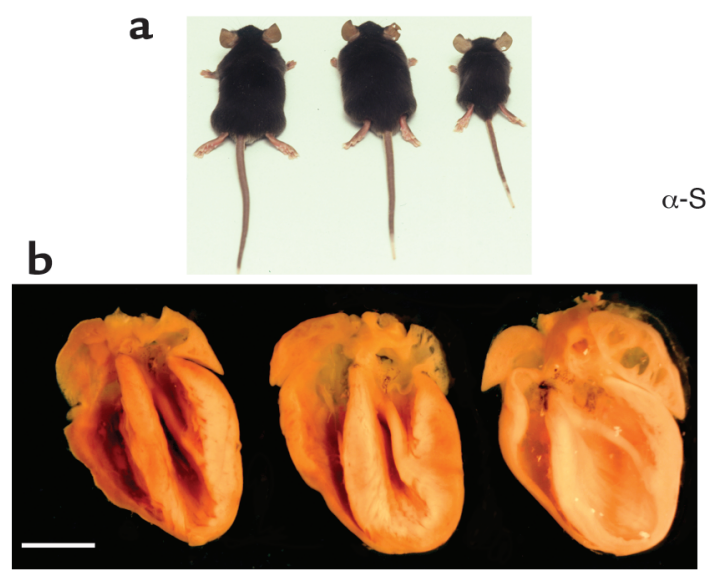

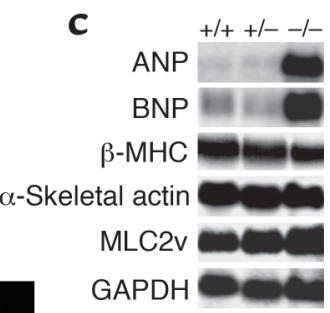



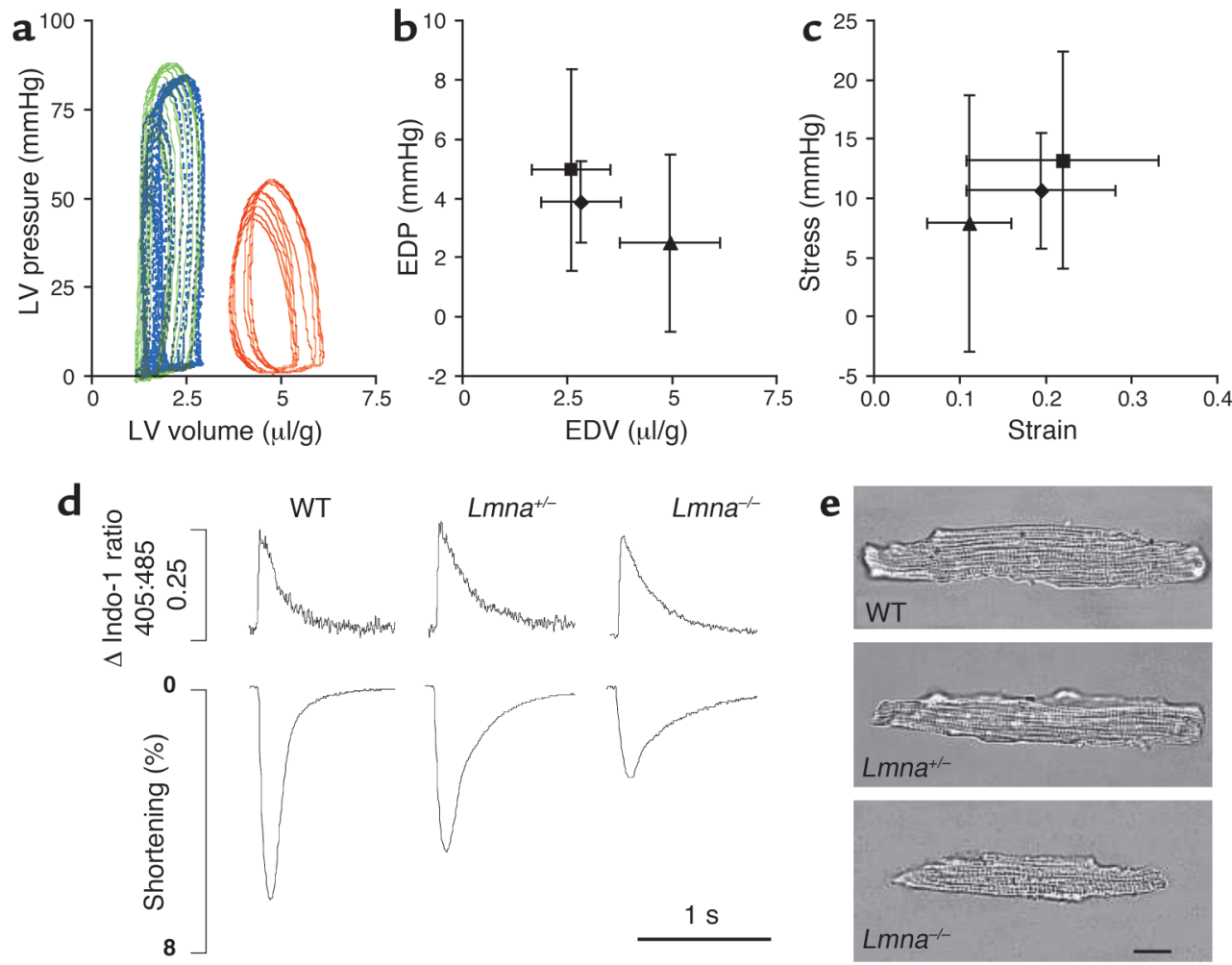

\section{Figure 2}

In vivo analyses of cardiac function with micromanometry and sonomicrometry in mice aged 4-6 weeks. (a) Representative LV pressure-volume loops recorded at steady state are shown for WT (blue), Lmna ${ }^{+/-}$(green), and $\mathrm{Lmna}^{-/-}$(red) mice. Steady-state LV end-diastolic pressure (EDP) plotted against LV end-diastolic volume (EDV) (b) and stress-strain relationships (c) for WT (diamonds, $n=9)$ Lmna ${ }^{+/-}$mice (squares, $n=11$ ), and $\mathrm{Lmna}^{-/}$- mice (triangles, $n=6$ ). Volume data are normalized to body weight; data are shown as mean \pm SD. Functional and morphologic characteristics of cardiac myocytes isolated from mice aged 4-6 weeks were also assessed. (d) Representative recordings of $\mathrm{Ca}^{2+}$ transients (top panel) and shortening (lower panel) in single myocytes. Intracellular $\mathrm{Ca}^{2+}$ concentration was measured as the change in the 405:485 nm emission ratio for Indo-1. 0.25 represents the magnitude of the $\mathrm{Ca}^{2+}$ transient. Lmna ${ }^{-/-}$myocytes have similar baseline and peak intracellular $\mathrm{Ca}^{2+}$ concentrations to WT and $\mathrm{Lmna}^{+/-}$myocytes but significantly reduced shortening. (e) $\mathrm{Lmna}^{-/-}$myocytes are shorter and thinner than WT and $L m n a^{+/-}$myocytes but the length/width ratios are similar. Scale bar: $20 \mu \mathrm{m}$.

observed in $L m n a^{-/-}$hearts was due to an intrinsic myocyte defect, the contractile properties of single LV myocytes were evaluated using an edge-detection technique. Consistent with the in vivo data, $L m n a^{-/}$ myocytes had reduced shortening compared with Lmna ${ }^{+/-}$and WT myocytes. Time to peak contraction and time to peak relaxation were prolonged in $\mathrm{Lmna}^{-/}$ and $\mathrm{Lmna}^{+/-}$myocytes (Table 3, Figure 2d). Evaluation of $\mathrm{Ca}^{2+}$ transients using Indo-1 fluorescence showed no differences in the baseline $\mathrm{Ca}^{2+}$ levels, peak transient amplitude, or time to peak amplitude between the three groups of mice; however, time to $50 \%$ relaxation of the $\mathrm{Ca}^{2+}$ transient was prolonged in $\mathrm{Lmna}^{-/-}$and $\mathrm{Lmna}^{+/-}$ myocytes (Table 3, Figure 2d). When myocyte fractional shortening was plotted against change in $\mathrm{Ca}^{2+}$, there was a significant reduction in the slope of the contraction phase in both $\mathrm{Lmna}^{-/}$and $\mathrm{Lmna}^{+/-}$myocytes when compared with WT mice, suggestive of altered $\mathrm{Ca}^{2+}$ affinity (Table 3). Evaluation of myocyte morphology showed that $L m n a^{-/-}$myocytes were relatively short and thin with reduced sarcomere length. The myocyte length/width ratio in the three groups was similar
(Table 3, Figure 2e). The latter finding was of particular interest, since development of eccentric hypertrophy with an increase in the length/width ratio is a characteristic feature of myocyte remodeling in DCM (33).

Selective changes in $L V$ expression of molecular markers of heart failure in Lmna-- mice. Sustained hemodynamic changes in DCM are associated not only with myocardial hypertrophy but also with transcriptional activation of immediate-early genes and reexpression of fetal genes (34). At 2 weeks of age, there were no changes in expression of any of the molecular markers of heart failure evaluated, including the immediate-early genes, c-fos, and c-jun. In $\mathrm{Lmna}^{-1-}$ mice aged 4-6 weeks, levels of ANP and BNP were increased (tenfold and 4-fold, respectively), with modest reductions of phospholamban (0.5-fold) and SERCA2a (0.2-fold) when compared with WT and $L m n a^{+/-}$mice (Figure 1c). Unexpectedly, given the presence of severe DCM, there were no changes in $\beta$-MHC or $\alpha$-skeletal actin.

Altered nuclear morphology and heterochromatin are prominent features of myocardial histopathology in Lmna/mice. Myocardial histological changes were present at 
Table 3

Morphology and function of cardiac myocytes isolated from mice aged 4-6 weeks

\begin{tabular}{|c|c|c|c|}
\hline & WT & $\mathrm{Lmna}^{+/-}$ & Lmna $^{-/-}$ \\
\hline \multicolumn{4}{|l|}{ Morphology and contraction } \\
\hline Myocyte length $(\mu \mathrm{m})$ & $178 \pm 9$ & $175 \pm 17$ & $144 \pm 17^{A, B}$ \\
\hline Myocyte width $(\mu \mathrm{m})$ & $32 \pm 4$ & $32 \pm 2$ & $26 \pm 3^{\mathrm{A}, \mathrm{B}}$ \\
\hline Sarcomere length $(\mu \mathrm{m})$ & $1.59 \pm 0.05$ & $1.55 \pm 0.05$ & $1.44 \pm 0.10^{\mathrm{A}, \mathrm{B}}$ \\
\hline Shortening (\%) & $6.47 \pm 2.95$ & $4.05 \pm 1.89$ & $3.76 \pm 1.82^{\mathrm{A}}$ \\
\hline Time to peak shortening (ms) & $70 \pm 11$ & $85 \pm 16^{A}$ & $113 \pm 28^{\mathrm{A}, \mathrm{B}}$ \\
\hline Time to $50 \%$ relaxation $(\mathrm{ms})$ & $73 \pm 16$ & $143 \pm 68^{A}$ & $193 \pm 124^{A}$ \\
\hline \multicolumn{4}{|l|}{$\mathrm{Ca}^{2+}$ transients } \\
\hline Baseline $\mathrm{Ca}^{2+}$ & $0.71 \pm 0.06$ & $0.70 \pm 0.11$ & $0.70 \pm 0.09$ \\
\hline Transient amplitude & $0.24 \pm 0.10$ & $0.24 \pm 0.06$ & $0.31 \pm 0.15$ \\
\hline Time to peak amplitude (ms) & $35 \pm 8$ & $36 \pm 8$ & $37 \pm 5$ \\
\hline Time to $50 \%$ relaxation $(\mathrm{ms})$ & $124 \pm 20$ & $184 \pm 55^{\mathrm{A}}$ & $205 \pm 82^{\mathrm{A}}$ \\
\hline Shortening-Ca ${ }^{2+}$ slope & $0.083 \pm 0.060$ & $0.025 \pm 0.008^{\mathrm{A}}$ & $0.021 \pm 0.014^{\mathrm{A}}$ \\
\hline
\end{tabular}

Cardiac myocyte properties were evaluated in WT $(n=8), \mathrm{Lmna}^{+/-}(n=9)$, and $\mathrm{Lmna}^{-/}$ $(n=9)$ mice. Myocyte dimensions were measured in up to 20 stored cell images from each mouse. Contractile function was estimated using edge detection. Cells were loaded with the $\mathrm{Ca}^{2+}$-sensitive fluorescent dye, Indo-1/AM, for simultaneous recording of intracellular $\mathrm{Ca}^{2+}$ concentration. Calibration of Indo-1 fluorescence was not performed due to potential problems associated with its compartmentalization within the cells and also additional fluorescence from incompletely de-esterified forms of the indicator. Values are therefore reported as relative changes in fluorescence ratio units following subtraction of background values. As an index of $\mathrm{Ca}^{2+}$-binding affinity, the relationship between myocyte shortening and change in $\mathrm{Ca}^{2+}$ concentration for each group of mice was plotted, and the slopes during contraction were compared. For each heart, four to six cells were studied. Averaged twitches and intracellular $\mathrm{Ca}^{2+}$ transients were constructed from a continuous sequence of 10-20 beats (see Methods). ${ }^{A} P<0.05$ compared with WT; ${ }^{B} P<0.05$ compared with $\mathrm{Lmna}^{+/-}$.

2 weeks of age and increased in severity by $4-6$ weeks of age (Figure 3). LV tissue from $\mathrm{Lmna}^{-/-}$mice had regions in which myofibrils had a stretched, wavy appearance, with occasional vacuolated cytoplasm and some increased interstitial spaces. There was no evidence of myofibril hypertrophy, necrosis, interstitial fibrosis, or altered amounts of ECM. Sarcomere organization and intercalated disc morphology appeared grossly normal in all mice studied (Figure 3, g-i, and data not shown). Alterations of nuclear morphology were prominent. WT myocyte nuclei had a well-rounded oval shape with a granular, dispersed pattern of chromatin and a distinct nucleolus (Figure $3 \mathrm{~d}$ ). $L m n a^{-/}$ myocytes contained nuclei that were elongated and lobulated with irregular, central chromatin clumps and a barely visible nucleolus (Figure 3f). $L m n a^{+/-}$nuclei had an intermediate phenotype (Figure 3e).

To further characterize these nuclear changes, myocardial sections were evaluated by immunofluorescence microscopy and electron microscopy (Figure 4). Although eccentric loss of lamin B staining at one pole has been described previously in $\mathrm{Lmna}^{-/-}$ embryonic fibroblasts (25), the nuclear lamina appeared intact in Lmna/- cardiomyocytes (Figure 4c). Hybridization with a pancentromeric heterochromatin probe confirmed that the dense deposits within Lmna ${ }^{-1-}$ myocyte nuclei were made up of heterochromatin and also demonstrated fragmentation and displacement of heterochromatin (Figure 4f). Electron microscopic images showed marked derangement of nuclear shape and size, together with peripheral and central heterochromatin clumping in Lmna ${ }^{-/-}$myocytes (Figure 4, i and l).

\section{Figure 3}

Myocardial histology in mice aged 4-6 weeks. Heart tissue from WT (a, d, and $\mathbf{g}), \mathrm{Lmna}^{+/-}(\mathbf{b}$, $\mathbf{e}$, and $\mathbf{h})$, and $\mathrm{Lmna}^{-/-}(\mathbf{c}, \mathbf{f}$, and $\mathbf{i})$ mice was evaluated by light microscopy after staining with H\&E (a-f) and electron microscopy $(\mathbf{g}-\mathbf{i})$. Cross-sections at the midventricular level (a-c) show LV and RV dilation in $\mathrm{Lmna}^{-/-}$hearts. Scale bar: $1 \mathrm{~mm}$. Higher magnification (d-f) shows overall myocardial architecture in $\mathrm{Lmna}^{-1-}$ LV myofibrils is relatively preserved. Nuclear elongation and chromatin dispersion are evident in $\mathrm{Lmna}^{+/-}$and $\mathrm{Lmna}^{-/-}$cardiomyocytes (insets). Scale bar: $50 \mu \mathrm{m}$. Sarcomere organization $(\mathbf{g}-\mathbf{i})$ appears normal in all mice. Scale bar: $2 \mu \mathrm{m}$.
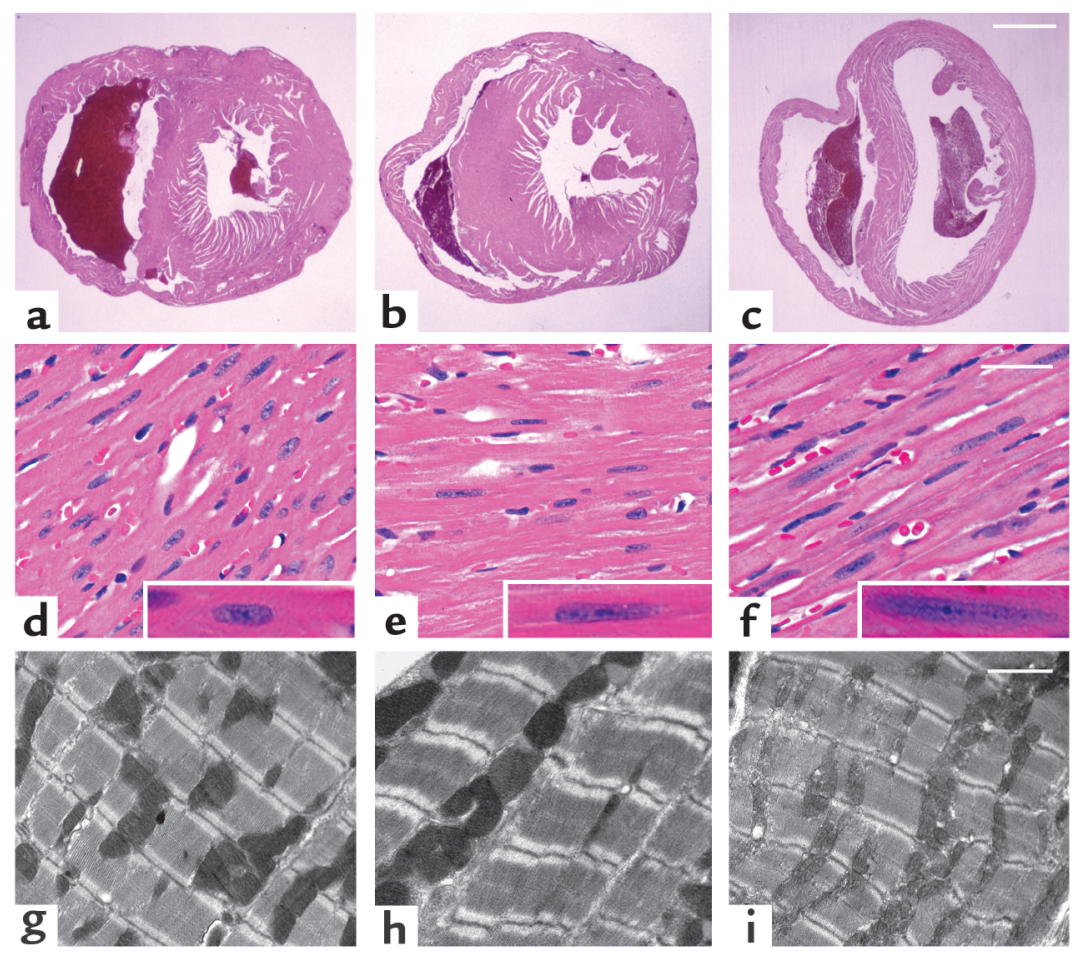

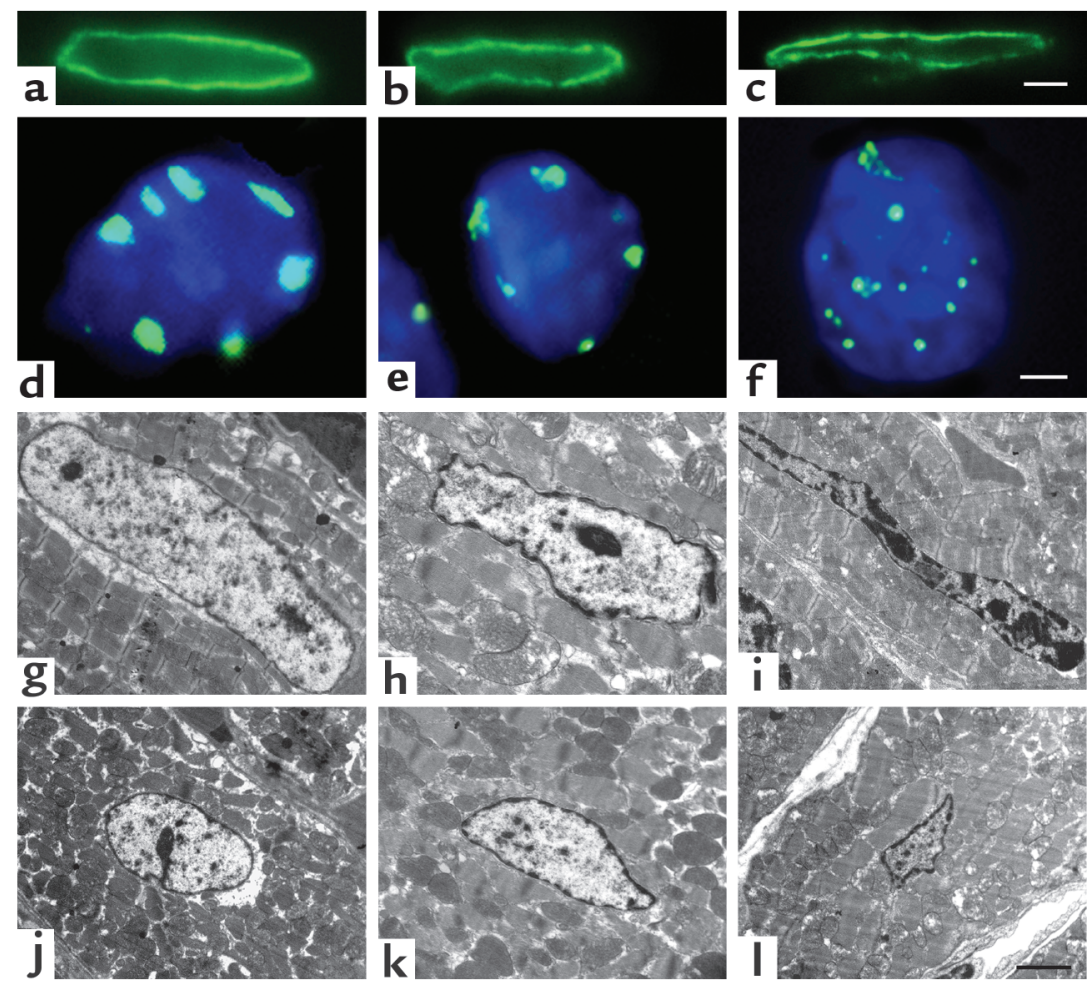

\section{Figure 4}

Nuclear morphology in LV cardiomyocytes from WT (a, d, g, and j), Lmna ${ }^{+/-}(\mathbf{b}, \mathbf{e}, \mathbf{h}$, and $\mathbf{k})$ and $\mathrm{Lmna}^{-/-}(\mathbf{c}, \mathbf{f}, \mathbf{i}$, and $\mathbf{I})$ mice aged 4-6 weeks. Immunostaining with a lamin B antibody $(\mathbf{a}-\mathbf{c})$ shows the nuclear lamina intact but altered in shape in $\mathrm{Lmna}^{+/-}$and $\mathrm{Lmna}^{-/-}$mice. Scale bar: $2 \mu \mathrm{m}$. Immunostaining with a pancentromeric heterochromatin probe (green) and DAPI (blue) (d-f) shows that centromeric heterochromatin forms discrete peripherally located blocks in WT nuclei with some irregularities of shape in $\mathrm{Lmna}^{+/-}$nuclei. In $\mathrm{Lmna}^{-/-}$nuclei, centromeric heterochromatin appears as small round foci at the periphery and within the nuclear interior. Confluent masses with a "beaded" appearance and larger, less dense masses with indistinct irregular borders are present. Scale bar: $1 \mu \mathrm{m}$. Longitudinal (g-i) and cross-sectional (j-I) electron microscopy images also demonstrate changes in nuclear morphology and heterochromatin organization. WT nuclei are characteristically oval-shaped with a thin rim of densely staining heterochromatin and a few small central deposits $(\mathbf{g}$ and $\mathbf{j})$. Lmna ${ }^{+/-}$nuclei have some irregularities of shape and peripheral heterochromatin clumping (h and $\mathbf{k})$. Abnormalities of shape and heterochromatin distribution are evident in $\mathrm{Lmna}^{-/-}$myocyte nuclei at 2 weeks of age (data not shown). By 4-6 weeks, the majority of $L m n a^{-/-}$myocyte nuclei demonstrate morphologic changes, including elongation and bizarre shapes with clumping of peripheral heterochromatin (i and I). In approximately $50 \%$ of the nuclei, heterochromatin clumps of varying size and shape are observed within the nuclear interior. Scale bar: $2 \mu \mathrm{m}$.

Apoptosis is a late manifestation of the disease phenotype in $\mathrm{Lmna}^{-/-}$mice. To determine whether apoptosis might be responsible for cardiac dysfunction and nuclear morphologic changes in $\mathrm{Lmna}^{-/-}$mice, serial evaluation of DNA fragmentation in LV tissue was performed using the TUNEL assay and DNA-laddering technique. Although there were no differences at 2 weeks, $\mathrm{Lmna}^{-/-}$mice had a significantly higher apoptotic index than WT and $L m n a^{+/-}$ mice at 4-6 weeks of age (Table 4). Similar results were obtained when DNA fragmentation was evaluated at 2 weeks and 4-6 weeks using the laddering technique.

Disruption of nuclear-desmin connections in lamin $A / C$-deficient mice. Since desmin filaments provide a direct physical link between the nuclear surface and the

Table 4

Apoptosis evaluation in mice aged 2 weeks and 4-6 weeks

\begin{tabular}{|c|c|c|c|c|c|c|}
\hline & \multicolumn{3}{|c|}{2 weeks } & \multicolumn{3}{|c|}{$4-6$ weeks } \\
\hline & WT & $\mathrm{Lmna}^{+/-}$ & Lmna $^{-1-}$ & WT & $L_{m n a^{+/-}}$ & $L^{2 m n a^{-1-}}$ \\
\hline TUNEL positive cells & $9.0 \pm 4.3$ & - & $8.3 \pm 2.3$ & $8.1 \pm 4.3$ & $5.9 \pm 4.7$ & $20.3 \pm 12.4$ \\
\hline Total myocytes & $7,081 \pm 288$ & - & $7,266 \pm 506$ & $3,955 \pm 775$ & $4,117 \pm 532$ & $4,238 \pm 768$ \\
\hline Apoptotic index (\%) & $0.13 \pm 0.06$ & & $0.11 \pm 0.03$ & $0.21 \pm 0.10$ & $0.14 \pm 0.12$ & $0.49 \pm 0.28^{\mathrm{A}, \mathrm{B}}$ \\
\hline
\end{tabular}

LV sections were evaluated for the presence of apoptosis by triple labeling using the TUNEL kit (to detect DNA fragmentation), DAPI (a DNA-specific stain used to identify nuclei), and anti- $\alpha$ sarcomeric actinin (to identify cardiomyocytes). Sequential images from 14-20 random fields were evaluated in a blinded fashion. The number of apoptotic nuclei was quantified using the apoptotic index, which was determined by dividing the number of positive-staining cardiomyocyte nuclei (derived from TUNEL and $\alpha$-actinin stains) by the total number of cardiomyocyte nuclei (derived from DAPI staining), expressed as a percentage. ${ }^{A} P<0.05$ compared with WT; ${ }^{B} P<0.05$ compared with $L_{m n a^{+/-}}$. 

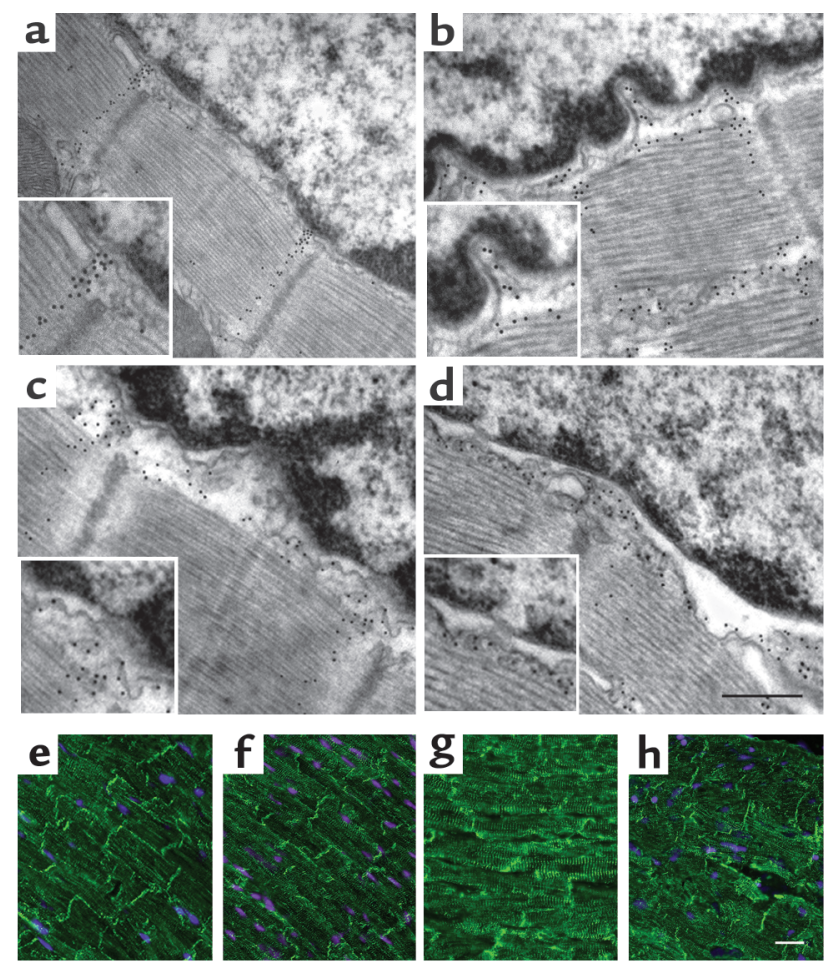

cytoskeleton, we speculated that disruption of the desmin filament network might be one mechanism by which nuclear changes could result in LV functional defects. The nuclear attachment points of the LV desmin filament network were evaluated in detail using immunogold electron microscopy (Figure 5, a-d). In WT mice, there was close proximity of the nuclear envelope and myofibril borders, which were bridged by welldefined desmin filament bands (Figure 5a). In contrast, $\mathrm{Lmna}^{-/}$mice had marked disorganization of the desmin filaments, dilation of the nuclear envelope space with distortion of the nuclear pores, and increased perinuclear space with regions in which the nucleus and adjacent cytoskeleton appeared to be completely disconnected. These changes were present at 2 weeks (Figure 5c) and increased in severity by 4-6 weeks (Figure $5 \mathrm{~d}$ ). $\mathrm{Lmna}^{+/-}$mice showed an intermediate extent of disorganization of desmin-nuclear attachments. Evaluation of the LV desmin cytoskeletal intermediate filament network using immunofluorescence microscopy showed progressive disorganization of the

\section{Figure 6}

Nuclear morphology and nuclear-desmin attachments in LA and skeletal myocytes in mice aged 4-6 weeks. Electron microscopic images of myocyte nuclei in the $L A(\mathbf{a}, \mathbf{b}, \mathbf{e}$, and $\mathbf{f})$ and gastrocnemius (c, $\mathbf{d}, \mathbf{g}$, and $\mathbf{h}$ ) show normal nuclear morphology and nuclear-desmin attachments in WT mice (a, c, e, and $\mathbf{g}$ ). Lmna ${ }^{-/-}$nuclei (b, d, f, and h) show changes in shape and heterochromatin clumping in the LA and skeletal muscle, although to a lesser extent than in the LV, with disruption and detachment of desmin filaments from the nuclear surface in both tissues. Scale bars: $2 \mu \mathrm{m}(\mathbf{a}-\mathbf{d})$ and $0.1 \mu \mathrm{m}(\mathbf{e}-\mathbf{h})$.

\section{Figure 5}

Disruption of the lamin-desmin intermediate filament network in lamin A/C-deficient mice. Attachment sites of desmin filaments to the LV cardiomyocyte nuclear surface were identified using immunogold-labeled desmin Ab's and electron microscopy. Approximately 30 nuclei from WT mice $(n=3), \mathrm{Lmna}^{+/-}$mice $(n=3)$, and $\mathrm{Lmna}^{-/-}$ mice $(n=3)$ aged 2 weeks and $4-6$ weeks were evaluated. In WT mice (a), desmin filaments (black dots) generally appear as well-defined bands connecting the cytoskeleton with the nuclear surface through nuclear pores (insets). Disorganization of the desmin filaments with detachment from the nuclear surface (with or without widening of the gap between the nuclear and myofibril borders) was observed in $4(13 \%) \mathrm{Lmna}^{+/-}$nuclei (b) and 16 (59\%) Lmna- ${ }^{-/-}$nuclei (d), but in none of the WT nuclei at 4-6 weeks. These changes were evident in 14 (50\%) $\mathrm{Lmna}^{-/-}$nuclei (c) at 2 weeks; scale bar $=0.25 \mu \mathrm{m}$. Immunostaining with a desmin Ab (e-h) shows normal Z disc striations and intercalated discs in WT and $\mathrm{Lmna}^{+/-}$mice, but progressive disorganization of the desmin filament network in 2-week-old $(\mathbf{g})$ and 4- to 6-week-old (h) $\mathrm{Lmna}^{-/-}$mice. Scale bar: $5 \mu \mathrm{m}$.

$Z$ disc cross-striation pattern, variation in the intensity of myofibril staining with patchy conglomerate formation, and regional diminution in the intensity of staining of intercalated discs in Lmna/- mice (Figure 5, $\mathrm{e}-\mathrm{h})$. Northern and Western blot analyses showed no differences in LV desmin RNA and protein levels, respectively, between the three groups of mice at 2 weeks or at 4-6 weeks of age. Altered nuclear morphology and disruption of nuclear-desmin attachment sites were observed also in the LA and skeletal muscle in Lmna-/- mice (Figure 6).

Progressive nuclear morphologic changes in Lmna-/- mice are associated with impaired nuclear translocation of SREBP1. Recently, a novel interaction between lamin A/C and SREBP1 was identified (35). SREBP1 is a transcription
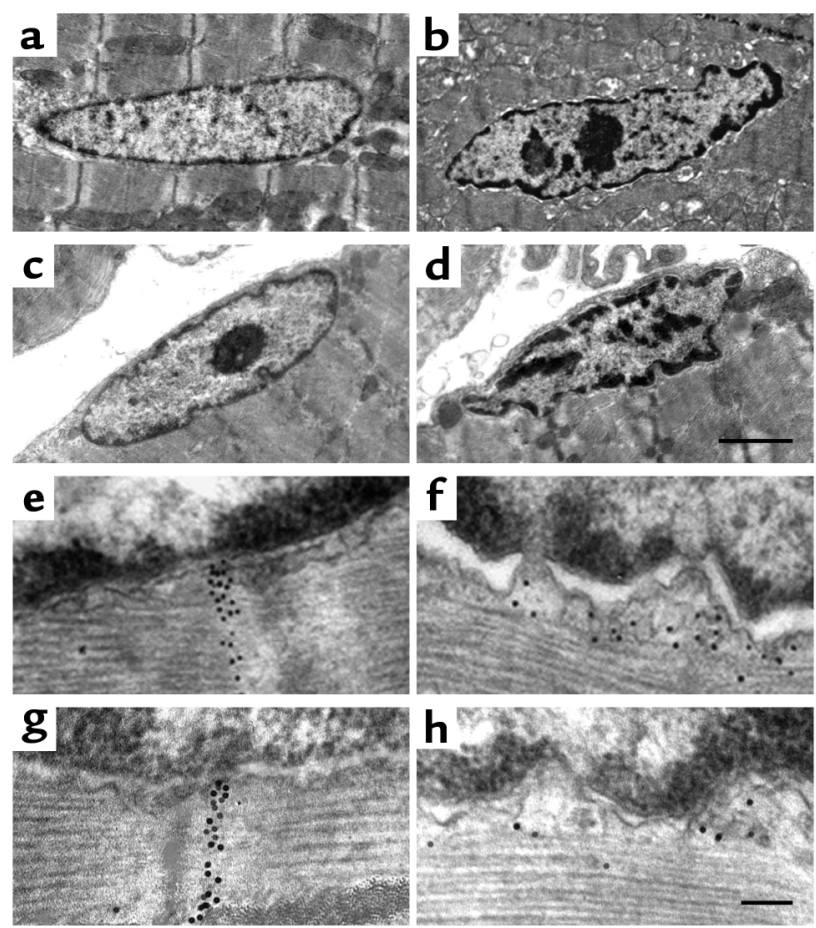


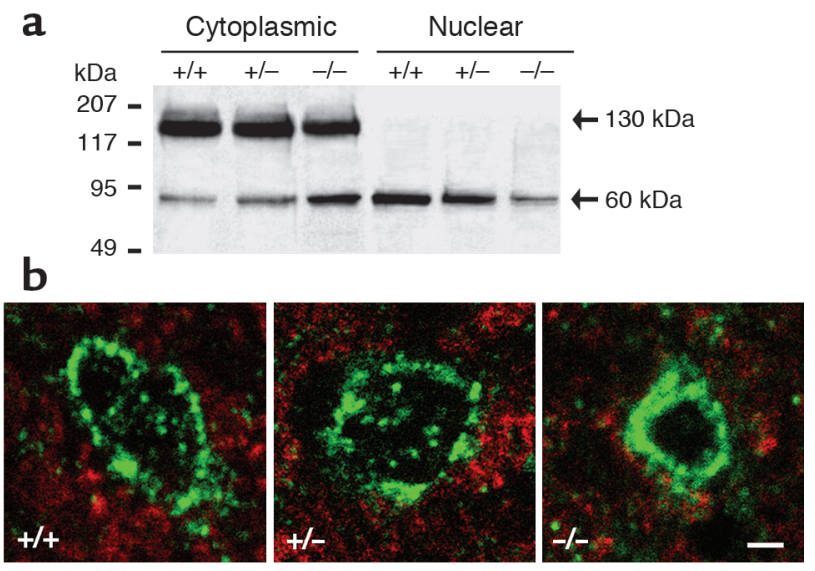

Figure 7

SREBP1 localization in LV cardiomyocytes of mice aged 4-6 weeks. (a) Western blot analysis of cytoplasmic and nuclear fractions of LV tissue hybridized with an SREBP1 Ab. Representative data for one WT (+/+), $\mathrm{Lmna}^{+/-}(+/-)$, and $\mathrm{Lmna}^{-/-}(-/-)$mouse are shown. Experiments were repeated with six mice from each group. When compared with WT cardiomyocytes, $\mathrm{Lmna}^{-/-}$cardiomyocytes show a mean decrease $(35 \%)$ in uncleaved $(130 \mathrm{kDa})$ SREBP1 and a mean increase $(55 \%)$ in cleaved $(60 \mathrm{kDa})$ SREBP1 in the cytoplasmic fraction, with a mean reduction (40\%) of cleaved SREBP1 in the nuclear fraction. (b) Immunofluorescence microscopy shows a perinuclear and intranuclear distribution of SREBP1 in WT and $\mathrm{Lmna}^{+/-}$nuclei, but predominant perinuclear SREBP1 staining in $\mathrm{Lmna}^{-/-}$nuclei. Scale bar: $2 \mu \mathrm{m}$.

factor of the basic helix-loop-helix family that regulates gene expression by binding to sterol regulatory elements in the nucleus (36). SREBPs are produced as membrane-bound precursors in the ER where they interact with the SREBP-cleavage-activating protein (SCAP). In lipoprotein-depleted cells, the SREBP-SCAP complex is translocated to the Golgi where it is cleaved by two proteases, releasing the $\mathrm{N}$-terminal domain that is translocated into the nucleus via nuclear pores. SREBPs have an important role in regulation of cholesterol and fatty acid synthesis in adipose tissue (36). Whether SREBPs are involved in metabolic homeostasis in other tissues, as well as the consequences of lamin A/C-SREBP1 interactions, are unknown.

We hypothesized that lamin A/C deficiency might alter the nuclear import and/or intranuclear actions of SREBP1 and hence contribute to the cardiac phenotype in $L m n a^{-/-}$mice. Evaluation of SREBP1 protein levels in cytoplasmic and nuclear fractions of LV tissue showed no differences at 2 weeks of age. In $L m n a^{-/-}$mice aged 4-6 weeks, the proportion of cleaved SREBP1 was increased in the cytoplasmic fraction and reduced in the nuclear fraction when compared with WT and $\mathrm{Lmna}^{+/}$ mice (Figure 7a). Immunostaining also demonstrated a predominant perinuclear distribution of SREBP1 in Lmna ${ }^{-/-}$LV tissue at 4-6 weeks (Figure 7b). Similar results were obtained in $\mathrm{Lmna}^{-/-}$LA tissue. There were no differences in LV or LA SREBP1 mRNA levels between WT, $L m n a^{+/-}$, and $L m n a^{-/-}$mice. Immunostaining of subcutaneous femoral and epididymal white adi- pose tissue and interscapular brown adipose tissue fragments showed no changes in SREBP1 distribution in $\mathrm{Lmna}^{-/-}$mice (data not shown). Since the intranuclear actions of SREBP1 in fatty acid metabolism are mediated by induction of PPAR $\gamma$ expression (37), levels of PPAR $\gamma$ protein in $\mathrm{LV}$ tissue were evaluated. In $\mathrm{Lmna}^{-1}$ mice, PPAR $\gamma$ protein levels were unchanged at 2 weeks, but were reduced $50 \%$ at 4-6 weeks when compared with WT mice (data not shown).

\section{Discussion}

Mutations in the LMNA gene have been shown recently to cause eight diverse human diseases, the pathophysiological basis of which has not been elucidated. We find that mice lacking lamin $\mathrm{A} / \mathrm{C}$ develop rapidly progressive DCM with conduction-system abnormalities. The critical defect arising from lamin $\mathrm{A} / \mathrm{C}$ deficiency is altered cardiomyocyte nuclear morphology, with changes in size, shape, and heterochromatin organization. Abnormalities of cardiac function appear to result predominantly from perturbation of cytoskeletal tension due to disruption of desmin attachment at the nuclearcytoskeletal interface. The absence of a compensatory hypertrophic response and impaired nuclear translocation of SREBP1 may contribute to disease progression and indicate that the dysmorphic cardiomyocyte nuclei are also functionally defective. Although young $\mathrm{Lmna}^{+/}$ mice have no overt cardiac phenotype, a 50\% reduction in lamin $\mathrm{A} / \mathrm{C}$ does elicit an intermediate extent of nuclear morphologic change and nuclear-desmin detachment. Long-term follow-up to determine the natural history of these mice is in progress. These data suggest a novel basis for defective force transmission in DCM and provide the first evidence for a disease mechanism in laminopathies.

Current concepts for the molecular pathogenesis of DCM. Identification of disease-causing genes has yielded significant insights into the molecular basis of familial DCM. Two pathophysiological mechanisms have been widely proposed: defective force transmission and defective force generation (reviewed in ref. 38). The cardiomyocyte cytoskeleton is made up of a series of interconnecting proteins that provide a direct physical link between the contractile apparatus and the ECM. Mutations in genes encoding cytoskeletal proteins are predicted to alter the structural properties or organization of components of this pathway, resulting in reduced efficiency of active force transduction, whereas mutations in genes that encode sarcomere or $\mathrm{Ca}^{2+}$-handling proteins impair actin-myosin interaction and force generation $(39,40)$. The discovery of mutations in the $L M N A$ gene raised intriguing questions about the relationship between lamin A/C and DCM, since lamins are not known to participate in force transmission or force generation in cardiac myocytes.

Lamin A/C deficiency alters cardiomyocyte nuclear morphology. Previous studies in various cell types have shown that lamins are required for maintenance of normal nuclear shape and size (reviewed in ref. 6). The 
basis for altered morphology has not been characterized precisely, but can be attributed to structural weakness of the lamina and loss of the intranuclear lamin $\mathrm{A} / \mathrm{C}$ scaffolding. Our data demonstrate that lamin A/C is a determinant of nuclear morphology in cardiomyocytes. In $\mathrm{Lmna}^{-/-}$mice, nuclear shape changes in the LV, LA, and skeletal muscle are more extensive than reported previously in hepatocytes (25), suggesting that structural defects in the lamina may render the nucleus more susceptible to deformation within a contractile tissue milieu. In addition, the elongation and bizarre shapes observed in LV nuclei suggest that longitudinal and radial forces generated by chamber dilation may further exacerbate the effects of lamin A/C deficiency. Although the consequences of altered shape and size have yet to be defined, adverse effects on nuclear organization and function are likely. Whether the predominance of muscle phenotypes associated with LMNA mutations might be explained in part by a greater degree of nuclear dysfunction in contractile tissues remains to be determined.

Lamin A/C deficiency and the "altered gene expression" hypothesis. Since lamins bind to heterochromatin and to various transcription factors, abnormal gene expression has been proposed as a potential disease mechanism for laminopathies. Lamin A/C deficiency has been shown previously to cause thinning and focal loss of heterochromatin around the nuclear rim in embryonic fibroblasts and adult hepatocytes (25). We find more severe heterochromatin disruption, with marked peripheral clumping and central displacement, in $\mathrm{Lmna}^{-/-}$cardiomyocyte nuclei. Immunofluorescence microscopy demonstrates fragmentation and disorganization of centromeric heterochromatin, indicating a critical role of lamin $\mathrm{A} / \mathrm{C}$ not only in anchoring heterochromatin at the nuclear periphery but also in maintaining heterochromatin architecture. Since heterochromatin location and architecture and the spatial arrangement of the nuclear matrix have been proposed to be important determinants of transcription regulation (41-45), altered gene expression would be expected in lamin A/C-deficient mice. Further studies are clearly required to determine temporal changes in gene expression and potential cause and/or effect relationships with DCM.

Lamin A/C deficiency and the "nuclear fragility" bypothesis. Several lines of evidence support the "nuclear fragility" hypothesis for laminopathies in which it is proposed that structural defects predispose the nucleus to rupture in response to mechanical stress with subsequent myocyte apoptosis. First, nuclear envelope herniation and rupture with osmotic stress have been observed in cell culture studies of LMNA mutations (20). Second, lamina disruption and heterochromatin clumping have been described as early features of apoptosis $(46,47)$. Third, exercise-induced apoptosis has been demonstrated in dystrophin-deficient skeletal muscle (48). Finally, apoptosis has been implicated in disease progression in DCM and in cardiac conduc- tion disorders (49-51). In Lmna/- cardiomyocytes, although nuclear morphologic changes were present at 2 weeks, DNA fragmentation was not identified. Since the process of apoptotic cell death is completed within 6 to 24 hours (52), evidence of DNA fragmentation would be expected if the nuclear morphologic changes were primarily caused by apoptosis. The presence of DNA fragmentation in Lmna $a^{-/}$hearts at 4-6 weeks is consistent with apoptosis as a consequence, rather than the cause, of impaired cardiac function. While these data do not support the nuclear fragility hypothesis as a primary disease mechanism in sedentary Lmna/- mice, they do not exclude the possibility that a minimal threshold level of mechanical stress might be required and hence, that "wear and tear" effects of repeated contractions could induce apoptosis in lamin A/C-deficient human hearts.

Lamin A/C deficiency causes detachment of desmin at the nuclear-cytoskeletal interface. A major finding of our study was evidence of disorganization and detachment of desmin filaments from the nuclear surface in lamin A/C-deficient mice. Desmin is a muscle-specific intermediate filament protein that forms a cytoskeletal scaffolding that provides strength and mechanical integrity to myocytes and has a putative role in cell signaling (53). Desmin filaments link myofibrils radially along $Z$ discs to the costameres, longitudinally to the intercalated discs, and attach directly to the nuclear surface. The desmin filament network interacts with lamin $B$ at the nuclear pores $(54,55)$. In vitro studies indicate that the binding affinity of lamin $B$ for desmin is enhanced in the presence of lamin A (56). In Lmna/- mice, lamin A/C-deficiency per se would be expected to impair lamin B-desmin binding. In addition, mechanical stresses imposed by distortion of nuclear shape, muscle contraction, and LV dilation could exacerbate the effects of weakened lamin B-desmin connections.

Nuclear-cytoskeletal discontinuity as a novel mechanism for defective force transmission in DCM. We propose that loss of nuclear anchoring with subsequent laxity of the desmin filament network and altered cytoskeletal tension would impair force transmission in LV cardiomyocytes and result in systolic contractile dysfunction. While nuclear detachment is likely to have initiated disorganization of the LV desmin filament network in $\mathrm{Lmna}^{-/}$mice, progressive degenerative changes in desmin filaments can also develop as a consequence of DCM (57). The extent to which perturbation of nuclear-desmin attachments might initiate DCM in humans requires further evaluation; however, the recent finding of desmin disorganization in cardiac biopsy tissue from an individual in whom DCM and conduction-system disease was caused by a missense LMNA mutation (24) is consistent with the hypothesis that these changes may be disease causing. Detachment of desmin filaments from the nuclear surface was also present in LA and skeletal myocytes in $\mathrm{Lmna}^{-/-}$mice. These observations suggest that perturbation of the 
muscle-restricted desmin intermediate filament network might be a generalized mechanism for muscle phenotypes associated with LMNA mutations.

Recently, a second lamin-related nuclear-cytoskeletal protein network was described. Lamin A/C has been shown to interact with the inner nuclear membrane proteins, nesprin-1 (also known as Myne-1 or Syne-1) and nesprin 2 (or Syne-2). These nesprins have now been shown to be short intranuclear isoforms of giant nesprin proteins that connect the nuclear envelope to the actin cytoskeleton (58-61). Loss of lamin $\mathrm{A} / \mathrm{C}$ binding to nesprin could exacerbate desmininduced changes in cytoskeletal tension. Additional studies are warranted to evaluate the nesprin-actin network in $\mathrm{Lmna}^{-/-}$mice.

Whether shorter sarcomere length in $\mathrm{Lmna}^{-/-}$mice represents growth retardation or disease pathophysiology has not yet been determined; hence, it is not known whether sarcomere function is normal or impaired. Abnormal sarcomere length could be associated with reduced systolic force generation mediated by alterations of $\mathrm{Ca}^{2+}$ release or binding affinity (62). Changes in intracellular $\mathrm{Ca}^{2+}$ release do not appear to account for differences in LV systolic function since the peak amplitude of $\mathrm{Ca}^{2+}$ transients was similar in the three groups of mice. While indirect estimates were consistent with abnormalities of myofilament $\mathrm{Ca}^{2+}$ binding affinity (Table 3 ), it is notable that equivalent changes were present in $\mathrm{Lmna}^{-/-}$and in $\mathrm{Lmna}^{+/-}$mice. Given the relatively normal fractional shortening in $\mathrm{Lmna}^{+/-}$myocytes, it is unlikely that altered $\mathrm{Ca}^{2+}$-binding affinity is a significant determinant of reduced contraction in Lmna ${ }^{-/-}$myocytes.

Lmna-1- mice have impairment of physiological hypertrophy. $\mathrm{Lmna}^{-/-}$mice have growth retardation from 2 weeks of age, manifested in the heart as small cardiomyocyte size. The basis for growth retardation in these mice has not been fully explained, but it is notable that the recently described homozygous $L m n a^{L 530 P / L 530 P}$ mutant mice also show growth retardation, small hearts, and small cardiomyocytes (63). These observations, and the normal size of $\mathrm{Lmna}^{+/-}$mice, indicate that a threshold level of up to $50 \%$ normal lamin $\mathrm{A} / \mathrm{C}$ function is required for physiological growth. Although cardiac function in Lmna ${ }^{L 530 P / L 530 P}$ mice has not been reported, the differences in heart size between these two mouse models may be explained by relatively greater impairment of $\mathrm{LV}$ contraction and/or less compensatory myocardial remodeling in $\mathrm{Lmna}^{-/-}$mice. The consequences of small myocytes in dilated hearts have been evaluated in detail in transgenic mice overexpressing the myelin basic protein kinase, mammalian sterile 20-like kinase 1 (64). Increased LV diameter and wall thinning in these mice were attributed to altered interactions between myocytes and lateral myocyte slippage. Myocyte growth retardation may have similar effects in $\mathrm{Lmna}^{-/-}$mice.

Lmna $^{-/-}$mice fail to develop compensatory LV bypertrophy. In addition to impairment of physiological hypertrophy, Lmna $a^{-/-}$mice have defective pathological hypertro- phy. Failing hearts undergo progressive LV remodeling that includes the development of myocardial hypertrophy and fibrosis (33). These structural changes in the LV walls, taken together with altered LV geometry, increase myocardial stiffness and LV end-diastolic pressure. Despite severe DCM, $\mathrm{Lmna}^{-/}$mice fail to develop a compensatory hypertrophic response, as evidenced by the absence of myocyte hypertrophy and lack of induction of the molecular markers, $\beta$-MHC and $\alpha$-skeletal actin. In the absence of hypertrophy and interstitial fibrosis, myocardial wall stiffness was unchanged.

Desmin is not considered to be an important determinant of the passive elastic properties of LV myocardium (65), hence disruption of the desmin filament network would not be expected to impact significantly on LV diastolic properties. It is notable that desmin-null mice have severe disruption of cardiomyocyte architecture associated with degenerative changes, calcification, and interstitial fibrosis from the first week of life, with evidence of myocardial hypertrophy from 4 weeks of age $(66,67)$. Despite a relatively greater extent of myocardial ultrastructural change, desmin-null mice have a more benign course than $L m n a^{-/}$mice with the gradual onset of DCM in later adult life and a normal life span (68). These observations suggest that the absence of compensatory hypertrophy in $\mathrm{Lmna}^{-/-}$mice may accelerate disease progression.

Nuclear dysfunction as a cause of a "failed" bypertrophic response. The absence of a hypertrophic response to DCM is not a feature unique to $\mathrm{Lmna}^{-/-}$mice. For example, mice lacking muscle LIM protein (69) and also those lacking the muscle-specific integrin $\beta_{1}$-interacting protein, melusin (70), exhibit a blunted hypertrophic response to mechanical stretch due to defective sensing of the stretch stimulus. In $\mathrm{Lmna}^{-/-}$mice, there could be defects in intracellular signal transduction, nuclear translocation, and/or nuclear responsiveness to hypertrophic stimuli. The appropriate increases in LV expression of ANP and BNP in the setting of severe DCM suggest that mechanisms for sensing and transduction of mechanical stretch are intact. Although ANP and BNP can exert antihypertrophic effects (71), additional factors are likely to be involved. Reduced nuclear translocation of SREBP1, taken together with altered nuclear morphology and heterochromatin, suggest that defects in nuclear pore transport and/or transcription regulation of growth-promoting or growthinhibitory factors may be responsible.

Lamin A/C-SREBP1 interactions. Alterations of lamin A/C binding to SREBP1 have been proposed as a possible factor in the development of familial lipodystrophy (35). Whether lamin A/C-SREBP1 interactions might have a role in other laminopathies is unknown. The absence of changes in intranuclear SREBP1 and PPAR $\gamma$ in $L V$ tissue from mice aged 2 weeks indicates that (a) lamin A/C is not essential for the nuclear import or intranuclear actions of SREBP1 in the heart, and (b) disruption of lamin A/C-SREBP1 interactions does not represent a primary mechanism for DCM. 
We propose that reductions in intranuclear SREBP1 and PPAR $\gamma$ in $\mathrm{Lmna}^{-/-}$mice at 4-6 weeks of age are caused by acquired defects in nuclear pore transport in LV myocyte nuclei. The normal distribution of SREBP1 in adipose tissue nuclei is consistent with this hypothesis. $L m n a^{-/}$mice have been reported previously to have low serum glucose, insulin, and triglyceride levels (72). While this metabolic phenotype could be attributed to generalized defects in SREBP1 function, we suggest that growth retardation, muscular dystrophy, cardiac dysfunction, and possible reduced food intake are more likely causes. Defective myocardial energetics due to extranuclear accumulation of SREBP1 in the heart may exacerbate myocardial contractile dysfunction.

In $\mathrm{Lmna}^{-/-}$mice, altered nuclear shape, size, and intranuclear organization; dilation of the nuclear envelope space; and disruption of lamin B-desmin connections might promote changes in nuclear pore structure that would critically influence nuclear-cytoplasmic transport and, hence, a range of intranuclear functions. Dynamic changes in nuclear pore conformation have been reported to occur under conditions of cardiomyocyte stress (73). Further studies are required to characterize nuclear pore structure and function in lamin A/C-deficient mice and to determine the extent to which nuclear transport defects might be attributable to the absence of lamin $\mathrm{A} / \mathrm{C}$ or the presence of DCM.

\section{Acknowledgments}

This work was supported by the Sylvia and Charles Viertel Charitable Foundation, the National Health and Medical Research Council of Australia, the David Family, and the Thomas Family. We thank Robert Graham (Victor Chang Cardiac Research Institute), Don Chisholm and Greg Cooney (Garvan Institute of Medical Research), Paddy Mallon (National Centre in HIV Epidemiology and Clinical Research [NCHECR], University of New South Wales), David Jans (Monash University), and Marcel Borgers (Universiteit Maastricht) for helpful discussions regarding data analysis.

1. Hozak, P., Sasseville, A.M., Raymond, Y., and Cook, P.R. 1995. Lamin proteins form an internal nucleoskeleton as well as a peripheral lamina in human cells. J. Cell Sci. 108:635-644.

2. Barboro, P., et al. 2002. Unravelling the organization of the internal nuclear matrix: RNA-dependent anchoring of NuMA to a lamin scaffold. Exp. Cell Res. 279:202-218.

3. Manilal, S., et al. 1999. Distribution of emerin and lamins in the heart and implications for Emery-Dreifuss muscular dystrophy. Hum. Mol. Genet. 8:353-359.

4. Stuurman, N., Heins, S., and Aebi, U. 1998. Nuclear lamins: their structure, assembly and interactions. J. Struct. Biol. 122:42-66.

5. Burke, B., and Stewart, C.L. 2002. Life at the edge: the nuclear envelope and human disease. Nat. Rev. Mol. Cell Biol. 3:575-585.

6. Hutchison, C.J. 2002. Lamins: building blocks or regulators of gene expression? Nat. Rev. Mol. Cell Biol. 3:848-858.

7. Fatkin, D., et al. 1999. Missense mutations in the rod domain of the lamin A/C gene as causes of dilated cardiomyopathy and conductionsystem disease. N. Engl. J. Med. 341:1715-1724.

8. Bonne, G., et al. 1999. Mutations in the gene encoding lamin A/C cause autosomal dominant Emery-Dreifuss muscular dystrophy. Nat. Genet. 21:285-288.

9. Muchir, A., et al. 2000. Identification of mutations in the gene encoding lamins $\mathrm{A} / \mathrm{C}$ in autosomal dominant limb girdle muscular dystrophy with atrioventricular conduction disturbances (LGMD1B). Hum. Mol. Genet. 9:1453-1459.

10. Cao, H., and Hegele, R.A. 2000. Nuclear lamin A/C R482Q mutations in Canadian kindreds with Dunnigan-type familial partial lipodystrophy. Hum. Mol. Genet. 9:109-112.

11. Shackleton, S., et al. 2000. LMNA, encoding lamin A/C, is mutated in partial lipodystrophy. Nat. Genet. 24:153-156.

12. De Sandre-Giovannoli, A., et al. 2002. Homozygous defects in LMNA, encoding lamin $\mathrm{A} / \mathrm{C}$ nuclear-envelope proteins, cause autosomal recessive axonal neuropathy in human (Charcot-Marie-Tooth disorder type 2) and mouse. Am. J. Hum. Genet. 70:726-736.

13. Novelli, G., et al. 2002. Mandibuloacral dysplasia is caused by a mutation in LMNA-encoding lamin A/C. Am. J. Hum. Genet. 71:426-431.

14. Eriksson, M., et al. 2003. Recurrent de novo point mutations in lamin A cause Hutchinson-Gilford progeria syndrome. Nature. 423:293-298.

15. De Sandre-Giovannoli, A., et al. 2003. Lamin A truncation in Hutchinson-Gilford progeria. Science. 300:2055

16. Cao, H., and Hegele, R.A. 2003. LMNA is mutated in Hutchinson-Gilford progeria (MIM 176670) but not in Wiedemann-Rautenstrauch progeroid syndrome (MIM 264090). J. Hum. Genet. 48:271-274.

17. Chen, L., et al. 2003. LMNA mutations in atypical Werner's syndrome. Lancet. 362:440-445.

18. Ostlund, C., Bonne, G., Schwartz, K., and Worman, H.J. 2001. Properties of lamin A mutants found in Emery-Dreifuss muscular dystrophy, cardiomyopathy and Dunnigan-type partial lipodystrophy. J. Cell Sci. 114:4435-4445.

19. Raharjo, W.H., Enarson, P., Sullivan, T., Stewart, C.L., and Burke, B. 2001. Nuclear envelope defects associated with LMNA mutations cause dilated cardiomyopathy and Emery-Dreifuss muscular dystrophy. J. Cell Sci. 114:4447-4457.

20. Vigouroux, C., et al. 2001. Nuclear envelope disorganization in fibroblasts from lipodystrophic patients with heterozygous R482Q/W mutations in the lamin A/C gene. J. Cell Sci. 114:4459-4468.

21. Holt, I., Clements, L., Manilal, S., Brown, S.C., and Morris, G.E. 2001. The $\mathrm{R} 482 \mathrm{Q}$ lamin $\mathrm{A} / \mathrm{C}$ mutation that causes lipodystrophy does not prevent nuclear targeting of lamin $\mathrm{A}$ in adipocytes or its interaction with emerin. Eur. J. Hum. Genet. 9:204-208.

22. Favreau, C., et al. 2003. Expression of lamin A mutated in the carboxylterminal tail generates an aberrant nuclear phenotype similar to that observed in cells from patients with Dunnigan-type partial lipodystrophy and Emery-Dreifuss muscular dystrophy. Exp. Cell Res. 282:14-23.

23. Bechert, K., Lagos-Quintana, M., Harborth, J., Weber, K., and Osborn, M. 2003. Effects of expressing lamin A mutant proteins causing EmeryDreifuss muscular dystrophy and familial partial lipodystrophy in HeLa cells. Exp. Cell Res. 286:75-86.

24. Sebillon, P., et al. 2003. Expanding the phenotype of LMNA mutations in dilated cardiomyopathy and functional consequences of these mutations. J. Med. Genet. 40:560-567.

25. Sullivan, T., et al. 1999. Loss of A-type lamin expression compromises nuclear envelope integrity leading to muscular dystrophy. J. Cell. Biol. 147:913-919.

26. Glower, D.D., et al. 1985. Linearity of the Frank-Starling relationship in the intact heart: the concept of preload recruitable stroke work. Circulation. 71:994-1009.

27. Karunanithi, M., and Feneley, M.P. 2000. Single-beat determination of preload recruitable stroke work relationship: derivation and evaluation in conscious dogs. J. Am. Coll. Cardiol. 35:502-513.

28. Terracciano, C.M.N., and Macleod, K.T. 1997. Effects of lactate on the relative contribution of $\mathrm{Ca}^{2+}$ extrusion mechanisms to relaxation in guinea-pig ventricular myocytes. J. Physiol. 500:557-750.

29. Koetsier, P.A., Schorr, J., and Doerfler, W. 1993. A rapid optimized protocol for downward alkaline Southern blotting of DNA. BioTechniques. 15:260-262.

30. Lockard, V.G. 1992. A simple technique for post-embedding ultrastruc tural immunogold labelling. Ultrastruct. Pathol. 16:47-50.

31. Slinker, B.K., and Glanz, S.A. 1988. Multiple linear regression is a useful alternative to traditional analyses of variance. Am. J. Physiol 255:R353-R367.

32. Esposito, G., et al. 2000. Cellular and functional defects in a mouse model of heart failure. Am. J. Physiol. Heart. Circ. Physiol. 279:H3101-H3112.

33. Francis, G.S. 1998. Changing the remodeling process in heart failure: basic mechanisms and laboratory results. Curr. Opin. Cardiol. 13:156-161.

34. Sadoshima, J., and Izumo, S. 1997. The cellular and molecular response of cardiac myocytes to mechanical stress. Annu. Rev. Physiol. 59:551-557.

35. Lloyd, D.J., Trembath, R.C., and Shackleton, S. 2002. A novel interaction between lamin A and SREBP1: implications for partial lipodystrophy and other laminopathies. Hum. Mol. Genet. 11:769-777.

36. Yokoyama, C., et al. 1993. SREBP-1, a basic-helix-loop-helix-leucine zipper protein that controls transcription of the low density lipoprotein receptor gene. Cell. 75:187-197. 
37. Fajas, L., et al. 1999. Regulation of peroxisome proliferator-activated receptor $\gamma$ expression by adipocyte differentiation and determination factor 1 /sterol regulatory element binding protein 1: implications for adipocyte differentiation and metabolism. Mol. Cell. Biol. 19:5495-5503.

38. Fatkin, D., and Graham, R.M. 2002. Molecular mechanisms of inherited cardiomyopathies. Physiol. Rev. 82:945-980.

39. Kamisago, M., et al. 2000. Mutations in sarcomere protein genes as a cause of dilated cardiomyopathy. N. Engl. J. Med. 343:1688-1696.

40. Schmitt, J.P., et al. 2003. Dilated cardiomyopathy and heart failure caused by a mutation in phospholamban. Science. 299:1410-1413.

41. Getzenberg, R.H. 1994. Nuclear matrix and the regulation of gene expression: tissue specificity. J. Cell. Biochem. 55:22-31.

42. Felsenfeld, G. 1996. Chromatin unfolds. Cell. 86:13-19.

43. Cockell, M., and Gasser, S.M. 1999. Nuclear compartments and gene regulation. Curr. Opin. Genet. Dev. 9:199-205.

44. Francastel, C., Walters, M.C., Groudine, M., and Martin, D.I.K. 1999. A functional enhancer suppresses silencing of a transgene and prevents its localization close to centromeric heterochromatin. Cell. 99:259-269.

45. Lomvardas, S., and Thanos, D. 2002. Modifying gene expression programs by altering core promoter chromatin architecture. Cell. 110:261-271.

46. Kerr, J.F.R., Wyllie, A.H., and Currie, A.R. 1972. Apoptosis: a basic biological phenomenon with wide-ranging implications in tissue kinetics. Br. J. Cancer. 26:239-257.

47. Arends, M.J., and Wyllie, A.H. 1991. Apoptosis: mechanisms and roles in pathology. Int. Rev. Exp. Pathol. 32:223-254.

48. Podhorska-Okolow, M., et al. 1998. Apoptosis of myofibres and satellite cells: exercise-induced damage in skeletal muscle of the mouse. Neuropathol. Appl. Neurobiol. 24:518-531.

49. James, T.N., St. Martin, E., Willis, P.W., and Lohr, T.O. 1996. Apoptosis as a possible cause of gradual development of complete heart block and fatal arrhythmias associated with absence of the AV node, sinus node, and internodal pathways. Circulation. 93:1424-1438.

50. Narula, J., et al. 1996. Apoptosis in myocytes in end-stage heart failure. N. Engl. J. Med. 335:1182-1189.

51. Olivetti, G., et al. 1997. Apoptosis in the failing human heart. N. Engl. J. Med. 336:1131-1141

52. Saraste, A., and Pulkki, K. 2000. Morphologic and biochemical hallmarks of apoptosis. Cardiovasc. Res. 45:528-537.

53. Capetanaki, Y., Milner, D.J., and Weitzer, G. 1997. Desmin in muscle formation and maintenance: knockouts and consequences. Cell Struct. Funct. 22:103-116.

54. Georgatos, S.D., Weber, K., Geisler, N., and Blobel, G. 1987. Binding of two desmin derivatives to the plasma membrane and the nuclear envelope of avian erythrocytes: evidence for a conserved site-specificity in intermediate filament-membrane interactions. Proc. Natl. Acad. Sci. U. S. A 84:6780-6784.

55. Lockard, V.G., and Bloom, S. 1993. Trans-cellular desmin-lamin B intermediate filament network in cardiac myocytes. J. Mol. Cell. Cardiol. 25:303-309.

56. Georgatos, S.D., and Blobel, G. 1987. Lamin B constitutes an interme- diate filament attachment site at the nuclear envelope. J. Cell Biol. 105: $117-125$

57. Schaper, J., et al. 1991. Impairment of the myocardial ultrastructure in dilated cardiomyopathy. Circulation. 83:504-514.

58. Zhang, Q., et al. 2001. Nesprins: a novel family of spectrin-repeat-containing proteins that localize to the nuclear membrane in multiple tissues J. Cell. Sci. 114:4485-4498.

59. Mislow, J.M.K., Kim, M.S., Belt Davis, D., and McNally, E.M. 2001 Myne-1, a spectrin repeat transmembrane protein of the myocyte inner nuclear membrane, interacts with lamin A/C. J. Cell Sci. 115:61-70.

60. Zhang, Q., Ragnauth, C., Greener, M.J., Shanahan, C.M, and Roberts, R.G. 2002. The nesprins are giant actin-binding proteins, orthologous to Drosophila melanogaster muscle protein MSP-300. Genomics. 80:473-481.

61. Starr, D.A., and Han, M. 2002. Role of ANC-1 in tethering nuclei to the actin cytoskeleton. Science. 298:406-409.

62. Allen, D.G., and Kentish, J.C. 1985. The cellular basis of the length-tension relation in cardiac muscle. J. Mol. Cell. Cardiol. 17:821-840.

63. Mounkes, L.C., Kozlov, S., Hernandez, L., Sullivan, T., and Stewart, C.L 2003. A progeroid syndrome in mice is caused by defects in A-type lamins. Nature. 423:298-301.

64. Yanamoto, S., et al. 2003. Activation of Mst 1 causes dilated cardiomyopathy by stimulating apoptosis without compensatory ventricular myocyte hypertrophy. J. Clin. Invest. 111:1463-1474. doi:10.1172/JCI200317459.

65. Balogh, J., Merisckay, M., Li, Z., Paulin, D., and Arner, A. 2002. Hearts from mice lacking desmin have a myopathy with impaired active force generation and unaltered wall compliance. Cardiovasc. Res. 53:439-450.

66. Milner, D.J., Weitzer, G., Tran, D., Bradley, A., and Capetanaki, Y. 1996. Disruption of muscle architecture and myocardial degeneration in mice lacking desmin. J. Cell Biol. 134:1255-1270.

67. Thornell, L.-E., Carlsson, L., Li, Z., Mericskay, M., and Paulin, D. 1997. Null mutation in the desmin gene gives rise to a cardiomyopathy. J. Mol. Cell. Cardiol. 29:2107-2124.

68. Milner, D.J., et al. 1999. The absence of desmin leads to cardiomyocyte hypertrophy and cardiac dilation with compromised systolic function. J. Mol. Cell. Cardiol. 31:2063-2076.

69. Knoll, R., et al. 2002. The cardiac mechanical stretch sensor machinery involves a $\mathrm{Z}$ disc complex that is defective in a subset of human dilated cardiomyopathy. Cell. 111:943-955.

70. Brancaccio, M., et al. 2002. Melusin, a muscle-specific integrin $\beta_{1}$-interacting protein, is required to prevent cardiac failure in response to chronic pressure overload. Nat. Med. 9:68-75.

71. Molkentin, J.D. 2003. A friend within the heart: natriuretic peptide receptor signaling. J. Clin. Invest. 111:1275-1277. doi:10.1172/JCI200318389.

72. Cutler, D.A., Sullivan, T., Marcus-Samuels, B., Stewart, C.L., and Reitman, M.L. 2002. Characterization of adiposity and metabolism in Lmnadeficient mice. Biochem. Biophys. Res. Commun. 291:522-527.

73. Perez-Terzic, C., et al. 1999. Structural plasticity of the cardiac nuclear pore complex in response to regulators of nuclear import. Circ. Res. 84:1292-1301. 